Background: Sjögren's syndrome (SS) is a systemic autoimmune disease characterized by periductal lymphocytic infiltration of the salivary and lacrimal glands, which results in reduced secretory functions and oral and ocular dryness (1). In addition, patients often have extraglandular manifestations, such as interstitial pneumonia and interstitial nephritis, and the appearance of autoantibodies such as anti-Ro/SS-A and La/SS-B antibodies. Salivary gland ultrasonography (SGUS) is typically used to evaluate the findings of salivary glands; thus, we can evaluate the severity of salivary gland disorders due to SS using SGUS in real time (2).

Objectives: To identify clinical indices, including disease activity, associated with glandular involvement evaluated using SGUS in patients with SS.

Methods: We enrolled patients with SS $(n=115)$ and non-SS sicca subjects $(n=90)$ who visited Nagasaki University Hospital between 1995 and 2019. The patients' SS classifications were based on the 2002 American-European Consensus Group (AECG) SS classification criteria (3). The non-SS sicca subjects exhibited sicca symptoms but did not fulfill the AECG SS classification criteria. SGUS and clinical indices such as age, sex, the focus score (FS), sicca symptoms, the Saxon test results, Schirmer's test results, anti-SS-A/Ro antibody positivity, anti-SS-B/La antibody positivity, anti-centromere antibody (ACA) positivity, serum immunoglobulin G levels, and the clinical European League Against Rheumatism SS disease activity index were examined. The ultrasonography (US) score was calculated based on SGUS imaging (hypoechoic area, hyperechoic band, and irregular border) (4).

Results: The US score was significantly higher in patients with SS than that in non-SS sicca subjects. In addition, we found significant correlations between the US score and FS in patients with SS. Multivariate analysis revealed the FS, Saxon test positivity, and ACA positivity as the variables independently associated with the US score in patients with SS. These results were the same in the primary SS patient group $(n=96)$. Patients with ACA positivity had significantly higher US scores compared to those in patients with ACA negativity, whereas the FS was not significantly high. In addition, patients with ACA positivity had significantly greater positivity of hyperechoic bands than that in patients with ACA negativity.

Conclusion: This study indicated that ACA positivity, which is not reflected in sialadenitis of SS, is associated with the US score in patients with SS. These results suggest that US findings of patients with ACA positivity might show specific changes in salivary glands, such as fibrosis, and not only sialadenitis (5).

REFERENCES:

[1] Ramos-Casals M, Tzioufas AG, Font J. Primary Sjogren's syndrome: new clinical and therapeutic concepts. Ann Rheum Dis. 2005;64(3):347-54.

[2] van Ginkel MS, Glaudemans A, van der Vegt B, Mossel E, Kroese FGM, Bootsma $\mathrm{H}$, et al. Imaging in Primary Sjogren's Syndrome. J Clin Med. 2020;9(8).

[3] Vitali C, Bombardieri S, Jonsson R, Moutsopoulos HM, Alexander EL, Carsons SE, et al. Classification criteria for Sjogren's syndrome: a revised version of the European criteria proposed by the American-European Consensus Group. Ann Rheum Dis. 2002;61(6):554-8.

[4] Takagi Y, Nakamura H, Sumi M, Shimizu T, Hirai Y, Horai Y, et al. Combined classification system based on ACR/EULAR and ultrasonographic scores for improving the diagnosis of Sjogren's syndrome. PLoS One. 2018;13(4):e0195113.

[5] Nakamura H, Kawakami A, Hayashi T, Iwamoto N, Okada A, Tamai M, et al. Anti-centromere antibody-seropositive Sjögren's syndrome differs from conventional subgroup in clinical and pathological study. BMC Musculoskelet Disord. 2010;11:140.

Disclosure of Interests: None declared

DOI: 10.1136/annrheumdis-2021-eular.1110

\section{POS0719 ADVERSE OUTCOMES AND REHOSPITALIZATION AFTER DELIVERY AMONG WOMEN WITH SYSTEMIC LUPUS ERYTHEMATOSUS OR RHEUMATOID ARTHRITIS AND THEIR INFANTS}

J. Sabo ${ }^{1}$, N. Singh ${ }^{1}$, D. A. Crane ${ }^{2}$, D. R. Doody ${ }^{3}$, M. A. Schiff ${ }^{4,5,6}$, B. A. Mueller ${ }^{3,6}$ ${ }^{1}$ University of Washington, Division of Rheumatology, Seattle, United States of America; ${ }^{2}$ University of Washington, Department of Rehabilitation Medicine, Seattle, United States of America; ${ }^{3}$ Fred Hutchinson Cancer Research Center, Public Health Sciences Division, Seattle, United States of America; ${ }^{4}$ University of Washington, Department of Obstetrics and Gynecology, Seattle, United States of America; ${ }^{5}$ University of New Mexico, Albuquerque, Division of Epidemiology, Biostatistics \& Preventive Medicine, Albuquerque, United States of America; ${ }^{6}$ University of Washington School of Public Health, Department of Epidemiology, Seattle, United States of America

Background: Women with rheumatoid arthritis (RA) or systemic lupus erythematosus (SLE) have greater risk of adverse obstetric and birth outcomes than women without these conditions. Infant outcomes are less well-studied. It is unknown whether re-hospitalization after delivery occurs more often for affected mothers and their infants.

Objectives: We compared obstetric outcomes among women with and without RA or SLE, and birth outcomes among their infants. Maternal and infant rehospitalizations $<2$ years of delivery were also compared.

Methods: This population-based cohort study used linked birth-hospital discharge data from Washington State for 1987-2014. International Classification of Disease $9^{\text {th }}$ revision (ICD9) codes identified all women with RA (ICD9 714.X, 725.X) and SLE (ICD9 710, 710.0, 710.1) in the hospital discharge record at delivery, and a 10:1 comparison group of women without these codes. Analyses were restricted to singleton live births (1,223 RA; 1,354 SLE). Poisson regression with robust standard errors estimated relative risks (RR) and 95\% confidence intervals $(\mathrm{Cl})$ for selected outcomes, accounting for delivery year, maternal age, and parity.

Results: Many adverse outcomes were more common among RA and SLE cases than among comparison women. Preeclampsia occurred more often during pregnancies of women with RA (RR 1.42, 95\% $\mathrm{Cl} 1.17-1.71$ ) or SLE (RR 2.33, 95\% Cl 2.01-2.70), as did preterm rupture of membranes (PROM, RR $2.85,95 \% \mathrm{Cl} 2.20-3.72$ for RA; RR $3.28,95 \% \mathrm{Cl} 2.54-4.23$ for SLE) Cesarean deliveries were more common among nulliparous women in both groups (RR 1.32, 95\% Cl 1.18-1.48 for both conditions). Infants of women with RA or SLE were more likely to weigh $<2500 \mathrm{~g}$ (RR 2.08, 95\% Cl 1.72-2.52 for RA; RR 4.88, 95\% Cl 4.27-5.58 for SLE), be small for gestational age (RR $1.25,95 \% \mathrm{Cl} 1.07-2.50 ; \mathrm{RR} 2.30 ; 2.04-2.59$, respectively), delivered at $<32$ weeks gestation (RR 1.83, 95\% Cl 1.13-2.97; RR 5.13, 95\% Cl 3.75-7.01 respectively), and require neonatal intensive care unit admission (NICU, RR 1.89, 95\% Cl 1.56-2.30; RR 2.71, 95\% Cl 2.25-3.28, respectively). Infants of women with SLE were more likely to have a malformation (RR $1.46,95 \% \mathrm{Cl}$ 1.21-1.75) or die within 2 years (RR 2.11, 95\% Cl 1.21-3.67). Rehospitalization levels among both women with RA (RR 2.22; 1.62-3.04) and SLE (RR 2.78 $95 \% \mathrm{Cl} 2.15-3.59)$ were greatest $<6$ months of delivery and declined over time. Infants of women with SLE had increased rehospitalization $<6$ months (RR 1.64, 95\% Cl 1.36-1.98).

Conclusion: Consistent with prior literature, we found women with RA or SLE experienced many adverse outcomes. In our data, these included preeclampsia, PROM, and cesarean deliveries, with increased risks more notable among women with SLE. Infants of women with either condition were more likely to weigh $<2500 \mathrm{~g}$, be $<32$ weeks gestation, small for gestational age, and require NICU admission than infants of comparison women. Only infants of women with SLE had increased malformations. Maternal rehospitalization after delivery was more common in both groups; most marked at $<6$ months. Infant rehospitalizations were increased in both cohorts to a lesser extent. Close follow-up during this time period is crucial to minimize adverse outcomes.

Disclosure of Interests: Julianna Sabo: None declared, Namrata Singh: None declared, Deborah A. Crane: None declared, David R. Doody: None declared, Melissa A. Schiff: None declared, Beth A. Mueller Shareholder of: Household owns shares in AstraZeneca

DOI: 10.1136/annrheumdis-2021-eular.1127

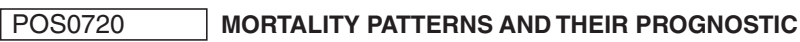 VALUE IN PATIENTS WITH SYSTEMIC LUPUS ERYTHEMATOSUS IN CUBA}

H. Hernández-Negrín ${ }^{1}$, Y. Roque-Dapresa ${ }^{2} .{ }^{1}$ Universidad de Ciencias Médicas de Villa Clara, Internal Medicine, Santa Clara, Cuba; ${ }^{2}$ Hospital Universitario Arnaldo Milián, Internal Medicine, Santa Clara, Cuba

Background: There is an increase over time in studies adopting cluster analysis (CA) to identify causes, patterns and severity in rheumatic diseases. ${ }^{1}$ However $\mathrm{CA}$ has not been used to identify patterns of causes-of-death in patients with systemic lupus erythematosus (SLE)

Objectives: 1) to identify homogeneous groups of patients according to their causes-of-death, 2) to identify homogeneous groups of cause-of-death combinations and 3) to determine the potential prognostic value of the identified patterns. Methods: This is a population-based study using the mortality register of Villa Clara (province of Cuba) between 1990 and 2019. Based on International Classification of Disease (ICD), Nine and Tenth Revision (ICD-9, code 710.0 and ICD-10, code M32), there were identified the SLE-related deaths (any mention on the death certificate) and their associated causes-of-death. Two hierarchical CA with Ward's method was carried out, based on the associated causes-ofdeath: one to conglomerate patients and another one to conglomerate variables. To identify differences in socio-demographic characteristics and causes-of-death between each cluster, Chi-square test, Fisher's exact test and Kruskal-Wallis tes were used as appropriate. Multivariate Poisson regression model corrected for over-dispersion was used to determine the prognostic value of the clusters. Years of potentially life lost (YPLL) was defined as a dependent variable. To calculate 
YPLL, each decedent's age at death was subtracted from a predetermined age of 75 years.

Results: A total of 141 patients were analyzed, which were grouped into three clusters. The socio-demographic features and causes-of-death that characterized each cluster are summarized in Table 1. Figure 1 shows the cause-of-death patterns identified by clustering the variables. After adjusting for sex, skin color, and the presence of pregnancy in the last year, cluster 1 was significantly associated with the number of YPLL in the multivariate analysis (adjusted rate ratios= $1.32 ; 95 \%$ confidence interval $=1.03$ to $1.70 ; p=0.028$ ).

Table 1. Socio-demographic characteristics and top ten causes-of-death according to clusters.

\begin{tabular}{lcccc}
\hline Variable & Cluster 1 & Cluster 2 & Cluster 3 & $P$ value \\
& $\mathrm{n}=40$ & $\mathrm{n}=71$ & $\mathrm{n}=30$ & \\
\hline
\end{tabular}

Age at death median (IR) $\quad 36(28.5$ to 50.2$) \quad 46(30.0$ to 56.0$) \quad 49(39.0$ to 63.2$) \quad 0.022$

Sex $n(\%)$

Male

Female

Skin color $\mathrm{n}(\%)$

White

Non White

Associated causes-of-death $\mathrm{n}(\%)$

Acute respiratory failure

Chronic kidney disease

Essential hypertension

Heart failure

Lupus nephritis

Multiple organ failure

Pericarditis

Pneumonia

Pulmonary embolism

Septicemia

$$
\begin{gathered}
4(28.6) \\
36(28.3) \\
\\
18(21.4) \\
22(38.6) \\
7(\%) \\
2(11.8) \\
31(96.9) \\
2(22.2) \\
3(25.0) \\
39(95.1) \\
2(18.2) \\
4(40.0) \\
5(14.7) \\
4(20.0)
\end{gathered}
$$

$8(57.1)$

63(49.6)

2(14.3)

28(22.0)

0.873

45(53.6)

21(25.0)

26(45.6)

$9(15.8)$

0.070

$6(35.3)$

$0(0)$
$6(66.7)$

6(66.7)

$9(75.0)$

2(4.9)

$5(45.5)$

$5(50.0)$

$6(17.6)$

$15(75.0)$

$0(0)$

\section{9(52.9)}

$1(3.1)$

(11.1) $\quad 0.734$

$0(0) \quad 0.093$

$0(0) \quad 0.000$

$4(36.4) \quad 0.496$

$1(10.0) \quad 0.569$

23(67.6) $\quad 0.000$

$\begin{array}{ll}1(5.0) & 0.043\end{array}$

14(77.8) $\quad 0.000$

YPLL: Years of potentially life lost. IR: interquartile rank.

Conclusion: Three clusters of patients were identified, the one with higher prevalence of causes-of-death expressing renal damage presented higher mortality burden. The patterns of causes-of-death were mainly shaped by renal, cardiovascular and infectious causes. Better understanding of the association among causes of death, improves our comprehension of SLE and facilitate its clinical management.

\section{REFERENCES:}

[1] Han L, Benseler SM, Tyrrell PN. Cluster and Multiple Correspondence Analyses in Rheumatology. Rheum Dis Clin N Am. 2018; 44:349-60. https://doi. org/10.1016/j.rdc.2018.01.013

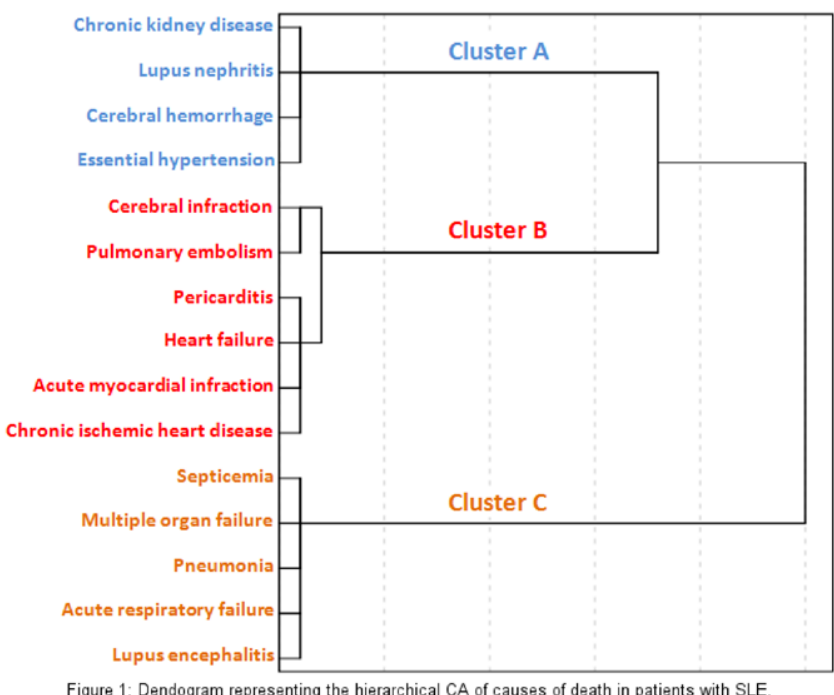

Disclosure of Interests: None declared

DOI: 10.1136/annrheumdis-2021-eular.1149

\section{\begin{tabular}{|l|l}
\hline POS0721 ARE ANTIMALARIALS SAFE FOR THE HEART \\
\hline
\end{tabular} OF PATIENTS WITH SYSTEMIC LUPUS ERYTHEMATOSUS? ANALYSIS OF FACTORS ASSOCIATED WITH THE DEVELOPMENT OF HEART FAILURE IN PATIENTS IN THE SPANISH SOCIETY OF RHEUMATOLOGY LUPUS REGISTRY (RELESSER)}

I. Rúa-Figueroa ${ }^{1}$, D. Rua-Figueroa ${ }^{2}$, A. M. Anzola Alfaro ${ }^{3}$, N. PérezVeiga $^{4}$, M. Galindo-Izquierdo ${ }^{5}$, J. Calvo-Alén ${ }^{6}$, A. Fernandez-Nebro ${ }^{7}$, R. Menor-Almagro ${ }^{8}$, C. Sanguesa ${ }^{9}$, E. Tomero Muriel ${ }^{10}, \mathrm{~N}$. Del-Val ${ }^{11}$,

E. Uriarte Isacelaya ${ }^{12}$, R. Blanco ${ }^{13}$, A. Boteanu ${ }^{14}$, J. Narváez $^{15}$, J. L. Andréu

Sánchez $^{16}$, T. Cobo-lbáñez ${ }^{17}$, C. A. Montilla-Morales ${ }^{18}$, M. Freire González ${ }^{19}$,

C. Bohórquez ${ }^{20}$, F. J. Toyos Sáenz de Miera ${ }^{21}$, E. Salas ${ }^{22}$, J. A. Bernal ${ }^{23}$,

E. Salgado Perez ${ }^{24}$, L. Expósito ${ }^{25}$, A. J. Mas $^{26}$, J. A. Hernandez Beriain ${ }^{27}$,

M. Velloso Feijoo ${ }^{28}$, N. Lozano Rivas ${ }^{29}$, G. Bonilla ${ }^{30}$, V. Quevedo Vila ${ }^{31}$,

M. Moreno ${ }^{32}$, I. Jiménez-Moleón ${ }^{33}$, O. Ibarguengoitia ${ }^{34}$, A. Pecondon ${ }^{35}$,

E. Aurrecoechea ${ }^{36}$, E. Valls-Pascual| ${ }^{37}$, C. Mouriño ${ }^{38}$, T. R. Vazquez

Rodriguez ${ }^{39}$, J. M. Pego-Reigosa ${ }^{38}$ on behalf of RELESSER and EAS-SER.

${ }^{1}$ Hospital de Gran Canaria Doctor Negrin, Rheumatology, Las Palmas GC, Spain; ${ }^{2}$ Hospital de Gran Canaria Doctor Negrin, Cardiology, Las Palmas GC, Spain; ${ }^{3}$ Hospital Gregorio Marañón, Rheumatology, Madrid, Spain; ${ }^{4}$ natalia.perez.veiga@gmail.com, Statistics, Las Palmas GC, Spain; ${ }^{5}$ Hospital Doce de Octubre, Rheumatology, Madrid, Spain; ${ }^{6}$ Hospital de Araba, Rheumatology, Vitoria, Spain; ${ }^{7}$ Hospital Carlos Haya, Rheumatology, Malaga, Spain; ${ }^{8}$ Hospital Jerez de la Frontera, Rheumatology, Jerez de la Frontera, Spain; ${ }^{9}$ Hospital Germán Trias i Pujol, Rheumatology, Barcelona, Spain; ${ }^{10}$ Hospital de la Princesa, Rheumatology, Madrid, Spain; ${ }^{11}$ Hospital de Navarra, Rheumatology, Navarra, Spain; ${ }^{12}$ Hospital Universitario Donostia, Rheumatology, San Sebastian, Spain; ${ }^{13}$ Hospital Marqués de Valdecilla, Rheumatology, Santander, Spain; ${ }^{14}$ Hospital Ramón y Cajal, Rheumatology, Madrid, Spain; ${ }^{15}$ Hospital de Belvitge, Rheumatology, Barcelona, Spain; ${ }^{16}$ Hospital Puerta de Hierro-Majadahonda, Rheumatology, Madrid, Spain; ${ }^{17}$ Hospital Infanta Sofia, Rheumatology, Madrid, Spain;

${ }^{18}$ Hospital de Salamanca, Rheumatology, Salamanca, Spain; ${ }^{19}$ Complejo Hospitalario Universitario de A Coruña (CHUAC), Rheumatology, A Coruña, Spain; ${ }^{20}$ Hospital Príncipe de Asturias, Rheumatology, Alcalá de Henares, Spain; ${ }^{21}$ Hospital Virgen Macarena, Rheumatology, Sevilla, Spain;

${ }^{22}$ Hospital Marina Baixa, Rheumatology, Villajoyosa, Spain; ${ }^{23}$ Hospital de Alicante, Rheumatology, Alicante, Spain; ${ }^{24}$ Complejo Hospitalario De Orense, Rheumatology, Orense, Spain; ${ }^{25}$ Hospital Universitario de Canarias, Rheumatology, Tenerife, Spain; ${ }^{26}$ Hospital Universitari Son Llàtzer, Rheumatology, Palma de Mallorca, Spain; ${ }^{27}$ Hospital Insular de Gran Canaria, Rheumatology, Las Palmas GC, Spain; ${ }^{28}$ Hospital de Valme, Rheumatology, Sevilla, Spain; ${ }^{29}$ Hospital Virgen de la Arrixaca, Rheumatology Murcia, Spain; ${ }^{30}$ Hospital La Paz, Rheumatology, Madrid, Spain; ${ }^{31} \mathrm{Hospital}$ de Monforte, Rheumatology, Lugo, Spain; ${ }^{32}$ Consorci Corporació Sanitària Parc Taulí, Rheumatology, Barcelona, Spain; ${ }^{33}$ Hospital Clínico San Cecilio, Rheumatology, Granada, Spain; ${ }^{34}$ Hospital de Basurto, Rheumatology, Bilbao, Spain; ${ }^{35}$ Hospital Miguel Servet, Rheumatology, Zaragoza, Spain;

${ }^{36}$ Hospital de Sierrallana, Rheumatology, Torrelavega, Spain; ${ }^{37}$ Hospital Universitario Dr. Peset, Rheumatology, Valencia, Spain; ${ }^{38}$ Galicia Sur Health Research Institute, Rheumatology, Vigo, Spain; ${ }^{39}$ Hospital Lucus Augusti, Rheumatology, Lugo, Spain

Background: Factors associated with the development of chronic heart failure (CHF) in systemic lupus erythematosus (SLE) have received little attention. On the other hand, recent data from the use of hydroxychloroquine in the treatment of SARS-CoV-2 infection during the COVID19 pandemic have cast some doubts on its cardiological safety.

Objectives: To identify factors associated to CHF in SLE.

Methods: Retrospective cross-sectional study, including all patients with SLE ( $\geq 4$ ACR-1997 criteria) recruited in RELESSER registry. The objectives and methodology of the registry have been described previously (1). CHF was defined according to the Charlson index item. Patients with CHF before diagnosis of SLE were excluded. Cumulative damage was measured with the SLICC/ ACR index, excluding cardiovascular (CV) items (mSDI). Multivariate analysis exploring factors associated with CHF was carried out.

Results: 117 patients ( $3 \%$ of the entire cohort) with SLE and CHF and 3,506 controls with SLE without CHF were included. $90 \%$ were women. Disease duration: mean (SD), 120.2 (87.7) months. CHF appeared after a median (P25-P75) of $9.40(4.2-18.3)$ years from SLE diagnosis. Patients with $\mathrm{CHF}$ were older $(59.8 \pm 18.2$ vs. $46.2 \pm 4.3)$. In the bivariate analysis, the 